\title{
Laser cooling using adiabatic rapid passage
}

\section{S. A. Malinovskaya}

Department of Physics, Stevens Institute of Technology, Hoboken, New Jersey, 07030, USA

E-mail: smalinov@stevens.edu

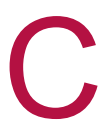
ooling and trapping of atoms and molecules is a prerequisite to studies of a multitude of quantum phenomena at ultracold temperatures. Early approaches to laser cooling rely on using monochromatic field in the scattering process of light by atoms into unoccupied modes of vacuum field with the rate of spontaneous emission as a time limiting factor. More recent cooling methods are based on stimulated process with polychromatic light and utilize adiabatic passage offering advantages of more frequent momentum exchanges with light and stronger cooling forces.

Stimulated Raman adiabatic passage (STIRAP) was used to generate ultracold molecules by transferring loosely bond Feshbach molecules to the fundamental ground state by applying two time-delayed ultrafast pulses in a counterintuitive sequence. Such an approach results in a creation of the dark state not involving a transitional state in the population transfer [1]. The two lasers were individually phase-locked to a femtosecond optical frequency comb. A direct implementation of a modulated optical frequency comb to transfer molecules from the Feshbach state to the ultracold state was proposed in Ref. [2]. In this work a sinusoidal modulation across each pulse in the pulse train induced a response in molecules analogous to the STIRAP resulted in the dark state formation and a stepwise population transfer to the ground state. The parity of the phase modulation was shown to play an important role in the controlled population transfer to the ultracold state [3]. In Ref. [4], a coherent train of weak pump-dump pairs of ultrashort shaped pulses was used for Raman transitions between the near-dissociation and the deeply bound vibrational levels to create ultracold molecules. The time shift between pulses equal to a half-period of vibration in the excited electronic state enabled damping of population to the final state without spontaneous emission losses.

Strong optical forces were produced on metastable $\mathrm{He}$ atoms by coherent control of momentum exchange between light via adiabatic passage using multifrequency light in Refs. [5, 6]. A carefully timed sequence of frequency-swept pulses produced a force much larger than the ordinary radiative force. The peak field intensity, the sweep direction and range, and the number of pulses were shown to be the effective control parameters in the scheme.

\footnotetext{
* Received April 1, 2021. This article can also be found at http://journal.hep.com.cn/fop/EN/10.1007/s11467-0211071-z.
}

Recently, a sawtooth-wave adiabatic passage (SWAP) scheme was demonstrated to cool atoms by rapidly sweeping the laser frequency of counter-propagating beams with Doppler shifts providing time-ordering to ensure photon recoils oppose the particle's motion [7-9]. This technique, when adopted to more complex multi-level systems, is shown to be resultative for cooling molecules [10]. The enhancement of the cooling force was achieved by applying a proper magnetic field decomposing the multi-level system into several two-level systems to provide an ordered stimulated absorption and emission allowing for a multiple photon momentum transfer. The approach in this work differs from the one in Ref. [8] in using the frequency ramp covering the entire hyperfine manifold and addressing all sublevels in each ramping process. The work suggests the solution for a weak Doppler cooling force problem relevant for multi-level type-II transitions in which the ground state total angular momentum quantum number is greater or equal to that of the excited state.

\section{References}

1. K.-K. Ni, S. Ospelkaus, M. H. G. de Miranda, A. Peér, B. Neyenhuis, J. J. Zirbel, S. Kotochigova, P. S. Julienne, D. S. Jin, and J. Ye, A high phase-space-density gas of polar molecules, Science 322, 231 (2008)

2. S. A. Malinovskaya and G. Liu, Harmonic spectral modulation of an optical frequency comb to control the ultracold molecules formation, Chem. Phys. Lett. 664, 1 (2016)

3. G. Liu and S. A. Malinovskaya, Creation of ultracold molecules within the lifetime scale by direct implementation of an optical frequency comb, J. Mod. Opt. 65, 1309 (2018)

4. A. Peér, E. A. Shapiro, M. C. Stowe, M. Shapiro, and J. Ye, Precise control of molecular dynamics with a femtosecond frequency comb, Phys. Rev. Lett. 101, 023601 (2008)

5. X. Miao, E. Wertz, M. G. Cohen, and H. Metcalf, Strong optical forces from adiabatic rapid passage, Phys. Rev. A 75,011402 (2007)

6. D. Stack, J. Elgin, P. M. Anisimov, and H. Metcalf, Numerical studies of optical forces from adiabatic rapid passage, Phys. Rev. A 84, 013420 (2011)

7. M. A. Norcia, J. R. K. Cline, J. P. Bartolotta, M. J. Holland, and J. K. Thompson, Narrow-line laser cooling by adiabatic transfer, New J. Phys. 20, 023021 (2018)

8. G. P. Greve, B. Wu, and J. K. Thompson, Laser cooling with adiabatic transfer on a Raman transition, New J. Phys. 21, 073045 (2019)

9. N. Petersen, F. Mühlbauer, L. Bougas, A. Sharma, D. Budker, and P. Windpassinger, Sawtooth-wave adiabatic passage slowing of dysprosium, Phys. Rev. A 99, 063414 (2019)

10. Q. Liang, T. Chen, W.-H. Bu, Y.-H. Zhang, and B. Yan, Laser cooling with adiabatic passage for type-II transitions, Front. Phys. 16(3), 32501 (2021) 